Louise Raissa Teixeira Viegas ${ }^{\text {a }}$

Milena Maria Cordeiro de Almeida ${ }^{\text {a }}$

a Instituto de Ciências da Saúde, Universidade Federal da Bahia, Salvador, BA, Brasil.

Contato:

Louise Raissa Teixeira Viegas

E-mail:

louiseviegas@hotmail.com

As autoras declaram que o trabalho não foi subvencionado e que não há conflitos de interesses.

O estudo é baseado em monografia de conclusão do curso de Fisioterapia, apresentada por Louise Raissa Teixeira Viegas, em novembro de 2014, no Instituto de Ciências da Saúde da Universidade Federal da Bahia.

Trabalho apresentado no Encontro Nordeste II, da Associação Brasileira da Rede Unida, Salvador (BA), em 2015.

\section{Perfil epidemiológico dos casos de LER/DORT entre trabalhadores da indústria no Brasil no período de 2007 a 2013}

\author{
Epidemiological profile of work-related musculoskeletal disorders \\ cases among industry workers in Brazil between 2007 and 2013
}

\section{Resumo}

Objetivo: descrever o perfil epidemiológico dos casos de lesões por esforço repetitivo (LER/DORT) entre trabalhadores da indústria no Brasil, notificados no período de 2007 a 2013. Métodos: estudo descritivo com dados secundários do Sistema de Informação de Agravos de Notificação (Sinan). Calcularam-se as proporções dos casos de LER/DORT de acordo com as variáveis do estudo, estimou-se o coeficiente de incidência (CI) das LER/DORT, total e por sexo, e calculou-se a variação proporcional percentual (VPP) no período estudado. Resultados: entre 2007 e 2013 foram notificados 17.537 casos de LER/DORT na Indústria, com um CI total de 7,4/100 mil trabalhadores em 2007 e de 16,7/100 mil em 2013, com uma VPP no período de 126\%. Os três principais diagnósticos foram: lesões no ombro (29,3\%), transtornos de sinóvias e tendões $(14,6 \%)$ e dorsalgias (14\%). Conclusão: diante do aumento das notificações, observa-se a importância do registro e análise dos casos para o planejamento e instituição de medidas de prevenção e atenção integral à saúde dos trabalhadores de acordo com as especificidades da atividade econômica desenvolvida por eles. Também é fundamental a discussão da responsabilidade das empresas e empregadores sobre o adoecimento dos trabalhadores.

Palavras-chave: doenças ocupacionais; indústrias; saúde do trabalhador; sistema de informações em saúde.

\begin{abstract}
Objective: to describe the epidemiological profile of reported industry work-related musculoskeletal disorders (WRMSD) in Brazil (2007-2013). Methods: descriptive study by secondary data analysis using the Notifiable Diseases Information System (Sinan) database. The proportion of WRMSD cases were calculated according to the study variables, the total and sex WRMSD incidence rates (IR) were estimated and the proportional percentage variance (PPV) throughout the studied period was reckoned. Results: 17,537 WRMSD industry cases were reported between 2007and 2013. They totaled an IR of 7.4/100,000 workers in 2007, increasing to 16.7/100,000 in 2013, with a PPV of $126 \%$. The three most common diagnoses were shoulder injuries $(29.3 \%)$, synovial and tendon disorders (14.6\%), and back pain (14\%). Conclusion: the notifications increase indicates the relevance of registering and analyzing the WRMDs cases in order to plan and introduce workers' preventive measures and comprehensive health care. Furthermore, the analysis of specific data, like the related economic activity, indicates the relevance of the discussion on the corporate and employers' responsibility over occupational diseases.
\end{abstract}

Keywords: occupational diseases; industry; worker's health; health information system. 


\section{Introdução}

As lesões por esforço repetitivo (LER/DORT) representam um importante agravo à saúde dos trabalhadores da indústria, tanto pelo número de notificações como pelo impacto na capacidade funcional e laboral dos indivíduos. Os centros industriais se destacam em relação ao elevado número de notificações de casos de LER/DORT, e isso se deve às exigências das atividades rigorosas impostas pelas indústrias, onde os trabalhadores são submetidos a um processo de trabalho caracterizado por jornadas de trabalhos extensas e sistemas de horas extras e são induzidos a competições para ganho de reconhecimento e aumento de produtividade ${ }^{1}$.

Os agravos relacionados ao trabalho, com o desenvolvimento das atividades econômicas industriais, apresentam-se como uma queixa decorrente de um desequilíbrio entre as exigências das tarefas relacionadas ao trabalho e as capacidades funcionais dos indivíduos. Dentre esses agravos, as Lesões por Esforço Repetitivo e Doenças Osteomusculares Relacionadas ao Trabalho (LER/DORT) se destacam pelo número de notificações e pelo impacto econômico e social, gerando dor, incapacidades, sofrimento e afastamentos do trabalho ${ }^{2}$.

Inúmeras categorias profissionais são atingidas pelo agravo denominado LER/DORT, nomenclaturas adotadas pelo Ministério da Saúde que definem esses agravos como fenômenos relacionados ao trabalho ${ }^{2}$.O principal agravo, responsável em quantidade de auxílios-doença e valor de auxílios-doença acidentários - cedidos pela Previdência Social entre 2011 e 2013 - são as doenças do sistema osteomuscular e do tecido conjuntivo. Apenas as causas externas estão adiante desses agravos $^{3}$. Na atividade econômica industrial, a partir da segunda metade do século XX, as LER/DORT adquiriram expressão em número e relevância social com a racionalização e inovação técnica na indústria $^{2}$. Atualmente, dentre as atividades econômicas, a indústria apresenta o maior número absoluto de diagnósticos de doenças do sistema osteomuscular e do tecido conjuntivo dentre os auxílios-doença acidentários concedidos pela Previdência Social no Brasil ${ }^{4}$. Além disso, publicações científicas também revelam a importante participação da indústria relacionada ao número de casos de LER/DORT no Brasil $^{2,5}$.

As LER/DORT são relatadas como lesões decorrentes do excessivo uso do sistema musculoesquelético, sem obter pausas para um tempo de recuperação ${ }^{5}$, sendo caracterizadas pela ocorrência de vários sintomas (concomitantes ou não), como dor crônica, parestesia, sensação de peso, fadiga muscular, que se manifesta principalmente no pescoço, cintura escapular e/ou membros inferiores ${ }^{6}$.

A quantidade de casos de LER/DORT vem aumentando anualmente, e esse fato explica-se pelas transformações do trabalho e das empresas que se organizaram de forma a visar a produtividade e lucro, desconsiderando, muitas vezes, os limites físicos e psicossociais dos trabalhadores. As altas exigências dos locais de trabalho, com alta demanda de movimentos repetitivos, a ausência de pausa, a permanência em determinadas posturas por tempo prolongado, além de equipamentos de trabalho desconfortáveis e sem ajustes necessários, repercutem negativamente na saúde dos trabalhadores ${ }^{7}$.

O reconhecimento, o diagnóstico e o registro das doenças relacionadas ao trabalho são regulamentados pela Portaria GM $777^{8}$, do Ministério da Saúde, de 28 de abril de 2004, que tornou de notificação compulsória vários agravos relacionados ao trabalho, dentre eles as LER/DORT, com operacionalização a partir de 2006. No Brasil, os dados provenientes dessas notificações compulsórias geram a base de dados do Sistema de Informação de Agravos de Notificação (Sinan), que tem como objetivo "o registro e processamento dos dados sobre agravos de notificação em todo território nacional, fornecendo informações para análise do perfil da morbidade e contribuindo, dessa forma, para a tomada de decisões em nível municipal, estadual e federal"11 . Os dados notificados são investigados por meio da aplicação da ficha de investigação "doença relacionada ao trabalho LER/DORT”, aplicada aos indivíduos por técnicos capacitados do estado. A finalidade da notificação é reconhecer e compreender melhor o problema, possibilitando o planejamento de ações de prevenção de novos casos e a promoção de estratégias de atenção à saúde dos trabalhadores ${ }^{8}$.

Vale ressaltar que apesar da instituição a Portaria Sinan em 2004, apenas em 2007 foi iniciada a implantação das notificações com os conteúdos da Saúde do Trabalhador no Sinan, especialmente após a Rede Nacional de Atenção Integral à Saúde do Trabalhador (Renast) investir em programas de treinamento, com cursos preparatórios das equipes das unidades sentinelas, serviços de saúde com a responsabilidade notificar estes agravos específicos ${ }^{9}$. Assim, reconhece-se e institucionaliza-se a importância da Vigilância em Saúde do Trabalhador, possibilitando uma atenção integral dos agravos e acidentes relacionados ao trabalho, desde a prevenção até a assistência, a partir do diagnóstico da situação de saúde dos trabalhadores brasileiros.

O objetivo deste trabalho foi investigar o perfil epidemiológico dos casos de LER/DORT entre trabalhadores da indústria no Brasil. 


\section{Métodos}

Trata-se de um estudo epidemiológico observacional, descritivo, com dados secundários, da população de trabalhadores da indústria acometidos por LER/DORT no Brasil. Foram utilizados dados do Sinan, abrangendo o período de 2007 a 2013.

Os dados estão em versão completa e atualizada, de 2006 a 2013, na base de dados de domínio público, concedida pelo Ministério da Saúde e que se encontra no site do Centro Colaborador de Vigilância dos Acidentes de Trabalho do Programa Integrado em Saúde Ambiental e Saúde do Trabalhador (Pisat/ Instituto de Saúde Coletiva/UFBA). Ressalta-se que a notificação dos casos de LER/DORT iniciou-se efetivamente em 2007, como descrito anteriormente, com os dados de 2006 representando um registro retrospectivo, optando-se por não utilizar dados desse primeiro ano.

O Sinan utiliza a ficha de investigação "doença relacionada ao trabalho LER/DORT" na construção da base de dados referida. Apesar da Portaria Sinan ${ }^{8}$ dispor sobre os procedimentos técnicos para a notificação compulsória de agravos à saúde do trabalhador em rede de serviços sentinela específica do Sistema Único de Saúde (SUS), todo e qualquer profissional de saúde pode realizar a notificação. Os Centros de Referência em Saúde do Trabalhador (Cerest), no âmbito dos territórios específicos, são polos irradiadores do conhecimento sobre a relação trabalho e saúde, com responsabilidade de oferecer suporte técnico e científico aos serviços SUS, em diferentes níveis de complexidade, além de deter a responsabilidade de notificar, investigar e dar respostas sensíveis aos agravos relacionados ao trabalho $^{9}$. Essas notificações relacionadas ao trabalho compõem o banco de dados Sinan, de livre acesso no Departamento de Informática do SUS (Datasus), além da disponibilização em centros colaboradores do Ministério da Saúde, como no acesso utilizado neste estudo, via centro colaborador Pisat/UFBA.

Este estudo utilizou a variável ocupação na seleção apenas dos casos de LER/DORT registrados para o grande grupo ocupacional de trabalhadores da produção de bens e serviços industriais da Classificação Brasileira de Ocupações $(\mathrm{CBO})^{10}$. A CBO foi utilizada para classificação da ocupação por esta apresentar menos perdas de registros, sub-registro, que a Classificação Nacional de Atividades Econômicas (CNAE).

Para o banco de dados gerado pelos casos de LER/ DORT, classificados como parte do grande grupo ocupacional indústria, foram analisadas as variáveis: sexo (feminino, masculino); faixa etária (18 a 35, 36 a 59, e 60 ou mais); raça/cor (brancos, não brancos); escolaridade (fundamental incompleto, ensino médio e ensino superior); e estado (UF, Brasil). Além disso, foram analisadas as variáveis ocupacionais: vínculo de trabalho (formal, informal); afastamento do ambiente de trabalho (sim, não); queixas de saúde (dor, diminuição de força muscular, limitação de movimento - todas com categorias sim ou não); afastamento do trabalho (sim, não); evolução do afastamento (melhora, piora); e evolução do caso (cura, incapacidade, temporária, incapacidade permanente total, outro). A classificação da faixa etária foi realizada a partir de uma reflexão sobre grupos de adultos jovens, adultos e idosos. Em relação às variáveis relacionadas às queixas de saúde, ressalta-se que elas não eram relatadas de forma exclusiva, uma vez que os indivíduos poderiam relatar um ou todos sintomas. As variáveis emissão da CAT (sim, não) e tipos de diagnósticos entre os casos de LER/ DORT (Classificação Internacional de Doenças, CID 10) também foram descritas.

Foram calculadas as proporções de casos de acordo com as variáveis do estudo e estimou-se a incidência das LER/DORT entre os trabalhadores da indústria. Para tanto, o número de casos registrados no Sinan representou o numerador e o número de trabalhadores da indústria, representado pela população economicamente ativa e ocupada (Peao) ${ }^{11}$ na atividade econômica indústria, incluindo a indústria da construção, registrado no Instituto Brasileiro de Geografia e Estatística (IBGE) representou o denominador. Para que o cálculo fosse realizado para todo o período do estudo, foram utilizados os dados da Peao da Pesquisa Nacional de Amostra de Domicílios (PNAD) para os anos de 2001 a 2009 e 2011 a 2013, e dos censos para 2000 e 2010. Como existe uma variação entre os dados registrados na PNAD e nos censos, a Peao expressa no Censo 2010 foi ajustada pelos valores da PNAD dos demais anos ${ }^{11}$. Os coeficientes foram multiplicados por uma base de 10 elevada à $5^{\text {a }}$ potência para ajuste dos valores.

Para avaliação da variação desses indicadores no tempo, foram calculadas as variações percentuais proporcionais (VPP) para cada indicador. Para tanto, considera-se a diferença entre os coeficientes de incidência (CI último ano investigado subtraído do CI primeiro ano investigado), dividida pelo CI do primeiro ano investigado e multiplicada por uma base de 100, o que representa a magnitude de variação percentual.

Os números de casos de LER/DORT no período estudado foram apresentados pelo período total, verificando assim a caracterização das variáveis estudadas. Devido ao sub-registro de algumas dessas variáveis, o cálculo foi baseado em 100\% dos dados registrados e não no total do número de notificações apresentadas pelo Sinan. 
A construção das informações foi realizada utilizando-se o programa Excel e o Epiinfo 3.5.4 (2012) e para a apresentação dos resultados utilizaram-se tabelas, gráficos e cartograma.

Este projeto foi aprovado pelo Comitê de Ética em Pesquisa da Escola de Enfermagem da Universidade Federal da Bahia, sob o número do Parecer 815.580 em 01/10/2014.

\section{Resultados}

Entre o período de 2007 a 2013, foram notificados no Sinan 17.537 casos de LER/DORT entre trabalhadores da indústria no Brasil, o que representa $47,9 \%$ das notificações desse diagnóstico para o período estudado. A proporção dos trabalhadores ativos e ocupados na indústria em relação às demais atividades econômicas variou de 36\% em 2007 e 34\% em 2013, ou seja, uma proporção inferior a $50 \%$ da Peao do Brasil para o período estudado.

A Peao total da indústria em 2007 era de 19.709 milhões de trabalhadores e em 2013, de 21.830 milhões, incluindo trabalhadores da Construção Civil. Para todo o período a Peao foi majoritariamente composta pelo sexo masculino, com um total de 14.702 milhões de trabalhadores homens em 2007 e de 16.899 milhões em 2013.

O coeficiente de incidência total no ano de 2007 foi de 7,4 por 100 mil trabalhadores, com um declínio apenas no ano seguinte e progredindo nos anos posteriores, atingindo 16,7 no ano de 2013, com uma variação proporcional percentual de $126 \%$.
O número de registros no sexo masculino foi de 1.014 em 2007 e 2.433 em 2013, com coeficiente de incidência crescente de 6,9 para 14,4 por 100 mil trabalhadores no período estudado. Isso representa uma variação proporcional percentual de 109\%. Na população feminina, o número de registros foi de 441 em 2007 e 1.201 em 2013, apresentando coeficiente de incidência em 2007 de 8,8/100 mil e no ano de 2013 de 24,4/100 mil trabalhadores, com uma variação proporcional percentual de 177\% (Gráfico 1).

$\mathrm{Na}$ análise das variáveis socioeconômicas, para todo o período investigado observou-se que, entre os casos notificados, a maioria era do sexo masculino, pertencia à faixa etária de 36 a 59 anos, apresentavam nível de escolaridade correspondente até ensino médio, raça/cor branca e vínculo de trabalho formal (Tabela 1).

Entre as variáveis que caracterizam o agravo e sua repercussão na saúde dos indivíduos, observou-se que $80,6 \%$ dos indivíduos apresentaram queixa de dor, $66,8 \%$ apresentaram limitação de movimento, 45,9\% queixas de diminuição de força muscular, $36,3 \%$ alterações de sensibilidade e $23,6 \%$ sinais flogísticos (Tabela 2). Foram observadas elevadas proporções de sintomas como limite ou incapacidade para o exercício de tarefas, além do afastamento das atividades laborais, sendo que dentre os trabalhadores que se afastaram do trabalho, 60,4\% evoluíram para a melhora. Ao se analisar a evolução geral dos casos de LER/DORT, observou-se que 55\% evoluíram com incapacidade temporária, 9,3\% obtiveram a cura e 6,7\% evoluíram para a incapacidade permanente. Dentre os casos registrados, 58,6\% tiveram a Comunicação de Acidente de Trabalho emitida (Tabela 2).

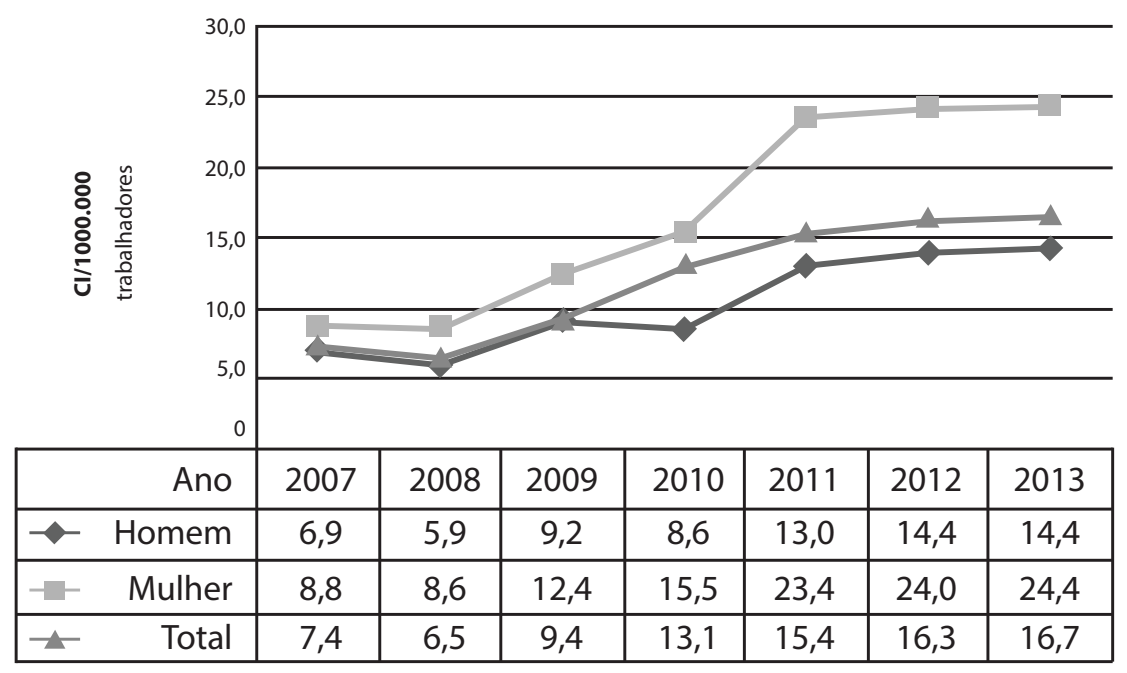

Gráfico 1 Coeficiente de incidência (CI) de casos de LER/DORT na indústria, total e por sexo - Brasil, 2007 a 2013. Fonte: Sinan, 2007-2013. Centro Colaborador da Vigilância aos agravos à Saúde do Trabalhador ${ }^{12}$ 
Tabela 1 Perfil socioeconômico dos trabalhadores da Indústria acometidos por LER/DORT no período de 2007 a 2013, Brasil.

\begin{tabular}{|c|c|c|}
\hline & $N=17.537$ & $\%$ \\
\hline \multicolumn{3}{|l|}{ Sexo } \\
\hline Masculino & 11.720 & 66,8 \\
\hline Feminino & 5.817 & 33,2 \\
\hline \multicolumn{3}{|l|}{ Faixa etária } \\
\hline 18 a 35 anos & 6.413 & 36,6 \\
\hline 36 a 59 anos & 10.706 & 61,0 \\
\hline 60 ou mais & 312 & 1,8 \\
\hline Outros/ignorados & 106 & 0,6 \\
\hline \multicolumn{3}{|l|}{ Escolaridade } \\
\hline Fundamental incompleto & 4.156 & 23,7 \\
\hline Médio & 8.990 & 51,3 \\
\hline Superior & 544 & 3,1 \\
\hline Ignorado/Não se aplica & 3.847 & 21,9 \\
\hline \multicolumn{3}{|l|}{ Raça/cor } \\
\hline Branco & 6.653 & 62,1 \\
\hline Não branco & 10.884 & 37,9 \\
\hline \multicolumn{3}{|l|}{ Vínculo de trabalho } \\
\hline Formal & 14.384 & 82,0 \\
\hline Informal & 2.208 & 12,6 \\
\hline Outros/Ignorados & 945 & 5,4 \\
\hline
\end{tabular}

Fonte: Sinan, 2007-2013. Centro Colaborador da Vigilância aos agravos à Saúde do Trabalhador ${ }^{12}$.

Os diagnósticos específicos mais frequentes dentre os registros do Sinan foram as lesões no ombro (CID 10: M75), os transtornos das sinóvias e tendões (CID 10: M65-68), as dorsalgias (CID 10: M54) e os transtornos dos discos vertebrais (CID 10: M50-53). Na classificação dos diagnósticos específicos de LER/ DORT, a categoria outros inclui diagnósticos menos frequentes e erros de classificação diagnóstica, como o exemplo de câncer de ovário e transtornos de ansiedade (Gráfico 2).

Entre as regiões brasileiras, a região Sudeste foi onde se observou o maior número de notificações, apresentando 11.028 casos no período investigado, sendo responsável por $62,9 \%$ dos casos no período. Em contrapartida, a região Centro-Oeste teve o menor número de notificações, apresentando 296 registros, com uma proporção de 1,7\% dos casos registrados entre 2007 a 2013 (Figura 1).
Tabela 2 Características dos casos de LER/DORT registrados em trabalhadores da Indústria, no período de 2007 a 2013 - Brasil.

\begin{tabular}{|c|c|c|}
\hline & $N=17537$ & $\%$ \\
\hline \multicolumn{3}{|l|}{ "Sinais e sintomas } \\
\hline Dor & 14.133 & 80,6 \\
\hline Limitação de movimento & 11.709 & 66,8 \\
\hline Diminuição de força & 8.057 & 45,9 \\
\hline Alteração de sensibilidade & 6.363 & 36,3 \\
\hline Sinais flogísticos & 4.132 & 23,6 \\
\hline \multicolumn{3}{|l|}{$\begin{array}{l}\text { Limite/incapacidade para } \\
\text { tarefas }\end{array}$} \\
\hline Sim & 13.798 & 78,7 \\
\hline Não & 1.682 & 9,6 \\
\hline Ignorado & 2.057 & 11,7 \\
\hline \multicolumn{3}{|l|}{ Afastamento } \\
\hline Sim & 12.181 & 64,5 \\
\hline Não & 3.656 & 20,8 \\
\hline Ignorados & 1.700 & 14,7 \\
\hline \multicolumn{3}{|l|}{ Evolução do afastamento } \\
\hline Melhora & 10.598 & 60,4 \\
\hline Piora & 4.004 & 22,8 \\
\hline Ignorados & 2.935 & 16,8 \\
\hline \multicolumn{3}{|l|}{ Evolução do caso } \\
\hline Incapacidade Temporária & 9.640 & 55,0 \\
\hline Cura - confirmada ou não & 1.634 & 9,3 \\
\hline Incapacidade Permanente & 1.178 & 6,7 \\
\hline Outros ou ignorados & 5.085 & 29,0 \\
\hline \multicolumn{3}{|l|}{ Emissão de CAT } \\
\hline Sim & 10.274 & 58,6 \\
\hline Não & 3.911 & 22,3 \\
\hline Não se aplica/ignorados & 3.352 & 19,1 \\
\hline
\end{tabular}

"Para "Sinais e sintomas" os números absolutos e relativos indicam a resposta sim para cada sinal/sintoma, por isso as proporções não somam $100 \%$.

Fonte: Sinan, 2007-2013. Centro Colaborador da Vigilância aos agravos à Saúde do Trabalhador ${ }^{12}$ 


\section{Outros}

Transt. Articulares (M23-25)

Exposição ocupacional

Transt. Tecidos Moles (M76-74)

Entesopatias (M76-79)

Mononeuropatias MMSS (G56)

Transt. Discos Vertebrais (M50-53)

Dorsalgias (M54)

Transt. Sinóvias/Tendões (M65-68)

Lesões no ombro (M75)

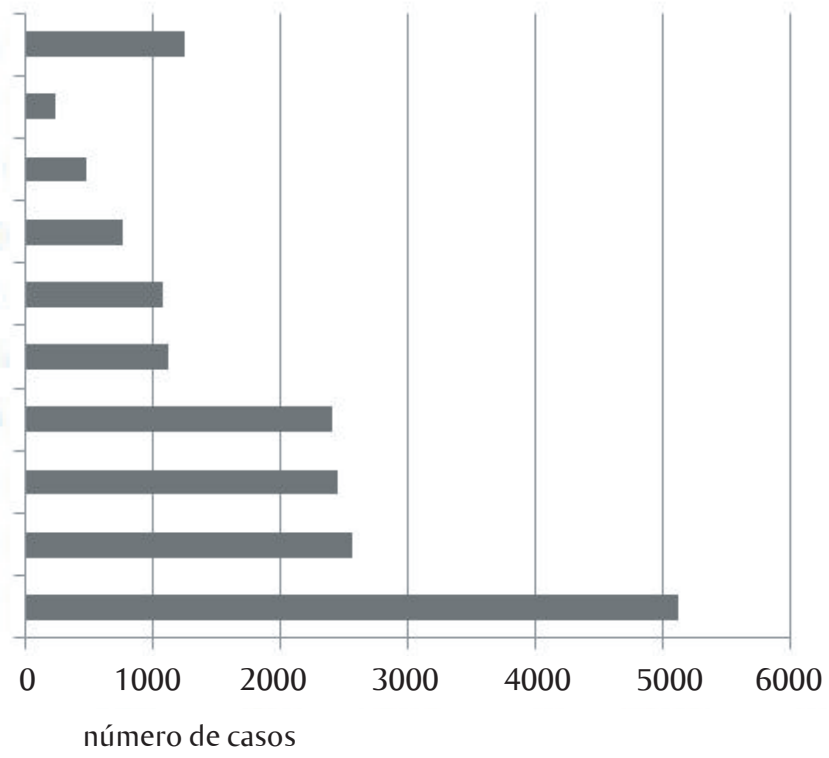

Gráfico 2 Concentração dos principais diagnósticos (CID 10) dentre os casos de LER/DORT na Indústria - Brasil, 2007 a 2013.

Fonte: Sinan, 2007-2013. Centro Colaborador da Vigilância aos agravos à Saúde do Trabalhador ${ }^{12}$

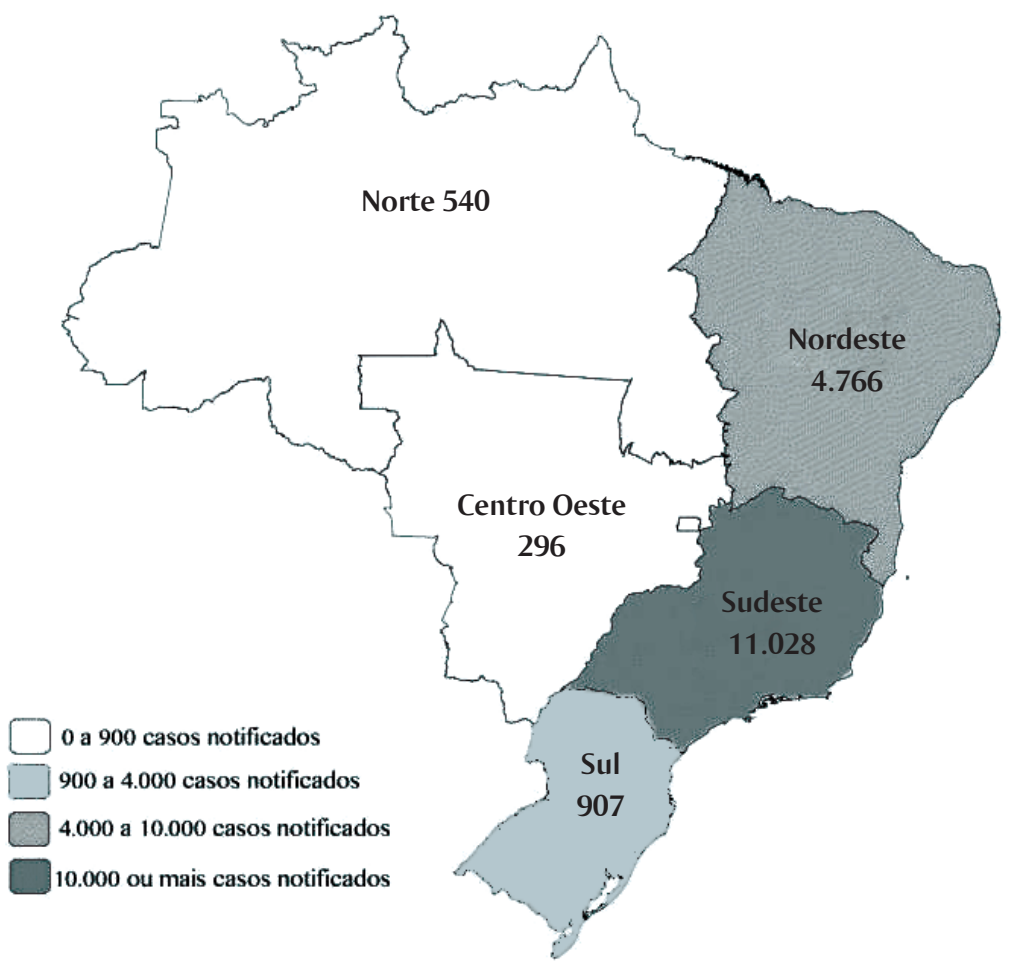

Figura 1 Distribuição de casos notificados de LER/DORT na indústria no Sinan, segundo a região geográfica-Brasil 2007 a 2013.

Fonte: Sinan, 2007-2013. Centro Colaborador da Vigilância aos agravos à Saúde do Trabalhador ${ }^{12}$ 


\section{Discussão}

A partir dos dados Sinan foi possível observar que o número de notificações de LER/DORT no setor industrial aumentou progressivamente nos últimos seis anos. Tal fato parece estar relacionado ao trabalho dos Cerests, que, a partir da instituição da Renast, têm ampliado sua capacidade de atuação, a partir do aumento do número de centros, de equipes e da descentralização de sua responsabilidade de suporte técnico e científico na relação saúde e trabalho para demais unidades do SUS, em especial para a Atenção Básica ${ }^{9}$. Assim, foi possível conhecer o perfil socioeconômico dos casos registrados de LER/DORT na indústria, além de observar a elevada prevalência de sintomas osteomusculares, como dor, limitação de movimento e diminuição de força muscular. A incidência desses sintomas pode ter influenciado diretamente o afastamento desses trabalhadores das atividades laborais, evoluindo também para incapacidades temporárias ou permanentes.

Como neste trabalho, um estudo realizado na indústria metalúrgica do Rio Grande do Sul encontrou um maior número de casos de LER/DORT no sexo masculino ${ }^{13}$. Tal fato pode ser justificado pela maior inserção de trabalhadores do sexo masculino nessa atividade econômica ${ }^{11}$. Porém, quando calculado o risco de adoecer nos trabalhadores da indústria neste trabalho, a população feminina apresenta um maior coeficiente de incidência quando comparada aos trabalhadores do sexo masculino. As mulheres estão normalmente inseridas nas ocupações com tarefas mais monótonas e repetitivas, além da composição corporal dessas ser caracterizada por menor massa muscular que os homens, o que pode representar fatores de risco para o sexo feminino, predispondo-as ao agravo ${ }^{13}$.

Tsuchiya, Mendonça e Cesar ${ }^{14}$, estudando a associação entre características pessoais, organização do trabalho e presença de dor em funcionários de uma indústria moveleira, encontrou um maior acometimento e maior relato de presença de dor entre as mulheres, o que foi justificado pela jornada dupla da mulher, maior repressão, gerando medo, tensão e estresse, além de as trabalhadoras geralmente serem responsáveis por trabalhos mais minuciosos.

Maciel, Fernandes e Medeiros ${ }^{15}$, em seu estudo sobre prevalência e fatores associados à sintomatologia dolorosa entre profissionais da indústria têxtil, ao analisarem a faixa etária de trabalhadores portadores de LER/DORT, relataram uma predominância dos casos em indivíduos na faixa de 20 a 39 anos, como observado nos casos registrados no Sinan. Para as LER/DORT, a idade pode estar relacionada ao acúmulo da exposição ao longo do tempo no trabalho e a redução fisiológica das capacidades funcionais. Porém, é importante salientar que tanto no referido estudo como nas informações obtidas dos dados do Sinan a população acometida pelas LER/DORT está em uma faixa etária adulta jovem, com grande potencial produtivo, o que pode expressar a carga das LER/ DORT afetando a vida produtiva e social de trabalhadores, com impacto econômico sobre seus familiares e Estado.

No estudo sobre distúrbios osteomusculares relacionados ao trabalho que observou o perfil de trabalhadores em benefício previdenciário em Diamantina, Minas Gerais, os resultados mostraram que há tendência de crescimento dos índices de afastamento por LER/DORT, sobretudo para os trabalhadores rurais, do setor industrial e de serviços públicos. Ainda nesse estudo, em relação ao nível de escolaridade, foi encontrada uma maior prevalência de casos nos indivíduos com ensino fundamental incompleto, observando-se também que os indivíduos com vínculo empregatício formal obtiveram maior acometimento ${ }^{16}$.

Fernandes et al. ${ }^{5}$ analisaram trabalhadores de indústria de plásticos e também encontraram um maior acometimento em trabalhadores com segundo grau completo. No estudo de Wang et al. ${ }^{17}$, com operadores de máquinas e costuras em indústrias de vestuários nos Estados Unidos, observaram que 45,2\% dos indivíduos acometidos não haviam completado o segundo grau. Assim, pode-se observar que o perfil socioeconômico dos casos de LER/DORT tende a variar dependendo do ramo industrial dos trabalhadores, uma vez que alguns desses ramos têm requisitos diferentes de escolaridade como critério de contratação, o que pode estar relacionado também com diferenças de faixa etária. Além disso, características como a raça/cor, variam dependendo da região do Brasil em que o ramo da atividade econômica industrial está inserido.

Observou-se neste estudo um maior número de notificações de trabalhadores no vínculo de trabalho formal da economia. Esse achado tem semelhança com outros estudos e evidencia que as estatísticas oficiais dos agravos relacionados ao trabalho no Brasil têm beneficiado os trabalhadores formais em detrimento dos informais ${ }^{18-21}$. Esse fato representa um problema, porque as notificações SUS deveriam avançar na proteção social dos trabalhadores seguindo o princípio da universalidade, ou seja, extrapolando a capacidade das notificações da Previdência Social, para além do reconhecimento dos agravos e acidentes dos trabalhadores formais. Além disso, reconhece-se que, majoritariamente, os postos de trabalho na indústria são caracterizados pelo vínculo formal e maiores níveis de escolaridade, em comparação com as ocupações da agricultura e serviços. 
Estudos epidemiológicos demostram que se registrou o aumento progressivo das LER/DORT no Brasil, tomando proporções epidêmicas, principalmente nas regiões de maior concentração industrial como São Paulo e Belo Horizonte, atingindo trabalhadores no auge de sua capacidade produtiva, coincidindo com a distribuição regional dos casos identificados neste trabalho ${ }^{22}$. Ressalta-se que essa concentração industrial do Sudeste produz casos de LER/DORT que extrapolam os limites territoriais dessa região, uma vez que muitos trabalhadores de outras regiões migram para o Sudeste em busca de emprego e renda. Observa-se, também, que as regiões mais desenvolvidas do Brasil apresentam melhor acesso a atenção à saúde, o que pode determinar uma melhor sensibilidade - e até qualidade - da notificação dos agravos relacionados ao trabalho.

Em relação aos efeitos desse adoecimento sobre a saúde dos trabalhadores, no estudo realizado em uma indústria metalúrgica do Rio Grande do Sul observou-se, por meio de questionários, proporções semelhantes às encontradas nas informações do Sinan: $75,2 \%$ dos trabalhadores relataram algum sintoma osteomuscular como dor, desconforto, dormência, diminuição de força muscular (sendo que metade desses indivíduos tiveram afastamento das atividades laborais devido aos sintomas ${ }^{13}$ ).

Dal Magro, Coutinho e Moré ${ }^{23}$, observaram no estudo sobre o obscurecimento da dor como dispositivo de controle da força de trabalho perante as LER/DORT, avaliando trabalhadores das indústrias de abate e processamento de carnes. Na maioria dos casos, as manifestações de dor descritas pelos entrevistados iniciaram de forma leve e esporádica, aumentando em frequência e intensidade e se alastrando para outros membros do corpo. Ao longo do tempo e diante da exposição contínua aos riscos laborais da atividade, muitos trabalhadores do setor passaram a conviver com a dor permanentemente ${ }^{23}$.

A evolução da dor aguda para um quadro de dor crônica também é um fator característico das LER/ DORT $^{24}$. Foi observado que alguns trabalhadores, antes de procurar auxílio nos serviços de saúde, criam formas de lidar momentaneamente com as dores e as dificuldades em saúde desencadeadas pelo trabalho. Observa-se mais demora nessa procura pelo serviço de saúde naqueles trabalhadores que têm medo de perder o emprego ${ }^{25}$. Essa convivência com a dor e a busca tardia por atenção à saúde, além de se expressar no sofrimento mental e físico desses trabalhadores, avança expressando-se na piora da situação de saúde dos trabalhadores, nas incapacidades temporárias, permanentes, absenteísmo e afastamentos.
Scussiato $^{26}$, ao analisar dentre os agravos relacionados ao trabalho as LER/DORT, os acidentes por material biológico e os acidentes grave notificados pela Unidade Saúde do Trabalhador, observou que todos os trabalhadores tiveram afastamento do serviço, variando entre dias, meses e anos. Com os dados obtidos sobre a evolução dos 20 (100\%) casos de LER/DORT, observou-se que apenas 5\% dos trabalhadores evoluíram para cura e 70\% sofreram incapacidade temporária. Esse resultado está em congruência com este artigo, que observou um maior percentual de indivíduos que evoluíram para incapacidade temporária. Segundo o autor, esse fato demonstra que a maioria dos trabalhadores que desenvolve LER/DORT sofre consequências temporárias e necessitam de longo período de acompanhamento multiprofissional para tratamento do agravo.

Todas essas informações desvelam a complexidade relacionada aos casos de LER/DORT, que afetam trabalhadores em idade economicamente ativa e produtiva, reduzindo o potencial transformador do trabalho, que, em vez de promover saúde, prazer, reconhecimento, autonomia, produção e reprodução social, gera dor, adoecimento e exclusão social.

No Brasil, a escassez e a inconsistência das informações sobre a real situação de saúde dos trabalhadores dificultam a definição de prioridades para as políticas públicas, o planejamento e a implementação das ações de Saúde do Trabalhador, além de privar a sociedade de subsídios importantes para a melhoria das condições de vida e trabalho desenvolvidos ${ }^{27}$.

Em relação aos sistemas de informação em saúde, ressalta-se o evidente sub-registro de agravos relacionados ao trabalho, uma vez que os números são incongruentes ao comparar distintos sistemas de informação - a exemplo do Anuário Estatístico da Previdência Social $^{3}$ e dados do Sinan, Ministério da Saúde ${ }^{8}$.

O estudo dos agravos relacionados ao trabalho tem se mostrado de importância fundamental para a Vigilância à Saúde, permitindo a realização do diagnóstico da ocorrência do evento na população, fornecendo subsídios para relações causais, indicando riscos, contribuindo, assim, para a análise da situação de saúde dos territórios. Em referência às diferenças de número de registros entre Sinan e Previdência Social, levanta-se o potencial do Sinan tanto na cobertura da população, independente do vínculo ou situação de trabalho, como na qualidade e variedade dos dados ${ }^{27}$.

\section{Conclusão}

Foi observado que o número de registros de LER/ DORT na indústria nos últimos seis anos aumentou 
progressivamente, mesmo diante do reconhecimento da subnotificação encontrada no Sinan, quando comparados com as informações da Previdência Social. Observa-se a grande importância do agravo devido a suas repercussões físicas, sociais e psicológicas e do reconhecimento do custo social dos adoecimentos e afastamentos, além do sofrimento, consequentes das LER/DORT, fazendo-se necessária a ampliação do olhar da saúde para a identificação, reconhecimento, registro e compreensão desse problema.
Uma vez reconhecido o problema, tornam-se possíveis medidas de prevenção e garantia de condições de trabalho que preservem a integridade física e psicológica dos trabalhadores, considerando as especificidades da atividade econômica desenvolvida por eles, além da discussão da responsabilidade das empresas e empregadores sobre o adoecimento dos trabalhadores. Assim, amplia-se o potencial do planejamento de ações de atenção integral à saúde desses trabalhadores, qualificando a atuação dos profissionais de saúde e proporcionando o estabelecimento de relação positiva entre o trabalho e a saúde.

\section{Contribuições de autoria}

Viegas LRT e Almeida MMC contribuíram na concepção, organização e delineamento do estudo, análise e interpretação dos resultados, redação e revisão crítica do conteúdo e aprovação da versão final do manuscrito.

\section{Referências}

1. Ghisleni PA, Merlo CRA. Trabalhador contemporâneo e patologias por hipersolicitação. Psicol Reflex Crit. 2005;18(2):171-6.

2. Maeno M, Salermo V, Rossi GAD, Fuller R. Lesões por Esforços Repetitivos (LER), Distúrbios Osteomusculares Relacionados ao Trabalho (DORT), Dor relacionada ao trabalho: Protocolos de atenção integral à Saúde do Trabalhador de Complexidade Diferenciada. Brasília, DF: Ministério da Saúde, 2006.

3. Brasil. Ministério da Previdência Social. Anuário Estatístico da Previdência Social 2013. Brasília, DF; 2014 [citado em 2015 maio 15]. Disponível em: http://bit.ly/2eK6o9X

4. Carvalho R. Distúrbios Musculoesqueléticos em Membros Superiores e Pescoço em Trabalhadores da Industria de Calçados [tese]. Salvador: Faculdade de Medicina da Universidade Federal da Bahia; 2013.

5. Fernandes RCP, Assunção AA, Silvany AM Neto, Carvalho FM. Musculoskeletal disorders among workers in plastics manufacturing plants. Rev bras epidemiol. 2010;13(1):11-20.

6. Chiavegato LG Filho, Pereira A Jr. Work related osteomuscular diseases: multifactorial etiology and explanatory models. Interface (Botucatu). 2004;8(14):149-62.

7. Kurionka I, Forcier L, Hagberg M, Silverstein B, Wells R, Smith MJ, et al. Works related musculoskeletal disorders (WMSDs): a reference book for prevention. London: Taylors \& Francis; 1995.

8. Brasil. Portaria $n^{0} 777 / G M$, de 28 de abril de 2004 . Dispõe sobre os procedimentos técnicos para a notificação compulsória de agravos à saúde do trabalhador em rede de serviços sentinela específica, no Sistema Único de Saúde - SUS. Brasília, DF; 29 abr 2004.

9. Galdino AG, Santana VS, Ferrite S. Os centros de referência em saúde do trabalhador e a notificação de acidentes de trabalho no Brasil. Cad. Saúde Pública. 2012;28(1):145-159.

10. Brasil. Portaria $n^{\circ} 397$, de 9 de outubro de 2002. Aprova a Classificação Brasileira de Ocupações $\mathrm{CBO} / 2002$, para uso em todo território nacional e autoriza a sua publicação. Ministério Trabalho e Emprego. Brasília, DF; 10 out 2002.

11. Instituto Brasileiro de Geografia e Estatística. Pesquisa Nacional por Amostra de Domicílios: pesquisa básica - 2001 a 2014. IBGE; 2013 [citado em 2015 maio 15]. Disponível em: http://bit. ly/29nCrxm

12. Centro Colaborador da Vigilância aos agravos à Saúde do Trabalhador. Sistema de Informação de Agravos de Notificação - Sinan. [citado em 2016 nov 22]. Disponível em: http://bit.ly/2gyD3ok

13. Picoloto D, Silveira DE. Prevalência de sintomas osteomusculares e fatores associados em trabalhadores de uma indústria metalúrgica de Canoas - RS. Ciênc saúde coletiva. 2008;13(2): 507-16.

14. Tsuchiya HZC, Mendonça CSL, Cesar ACG. Associação entre características pessoais, organização do trabalho e a presença de dor em funcionários de uma indústria moveleira. Fisioter Pesqui. 2009;16(4):294-8.

15. Maciel CCA, Fernandez BM, Medeiros SL. Prevalência e fatores associados à sintomatologia dolorosa entre profissionais da indústria têxtil. Rev bras epidemiol. 2006;9(1):94-102. 
16. Alcântara MA, Nunes SG, Ferreira SMCB. Distúrbios osteomusculares relacionados ao trabalho: o perfil dos trabalhadores em benefício previdenciário em Diamantina (MG, Brasil). Ciênc saúde coletiva. 2011;16(8):3427-36.

17. Wang PC, Rempel DM, Harrison RJ, Chan J, Ritz BR. Work-organizational and personal factors associated with upper body musculoskeletal disorders among sewing machine operators. Occup Environ Med. 2007;64(12):806-13.

18. García Gómez M, Castaneda Lopez RC. Desigualdades interterritoriales em La compensacion de las enfermedades profesionales em España de 1990 a 2007. Gac Sanit. 2009;23(5):373-9.

19. Souza NS, Santana VS. Incidência cumulativa anual de doenças musculoesqueléticas incapacitantes relacionadas ao trabalho em uma área urbana do Brasil. Cad Saúde Pública. 2011;27(11):2124-34.

20. Hennigton EA, Monteiro M. O perfil epidemiológico dos acidentes de trabalho no Vale dos Sinos e o sistema de vigilância em saúde do trabalhador. Hist cienc saude-Manguinhos. 2006;13(4):865-76.

21. Santana VS, Xavier C, Moura MCP, Oliveira R, Espírito-Santo JS, Araújo G. Gravidade dos acidentes de trabalho atendidos em serviços de emergência. Rev Saúde Publica. 2009;43(5):750-60.
22. Graup S. Cenário epidemiológico de morbidade no ambiente de trabalho no Brasil [tese]. Florianópolis: Universidade Federal de Santa Catarina, 2012.

23. Dal Magro MLP, Coutinho MC, Moré CLOO. O obscurecimento da dor como dispositivo de controle da força de trabalho frente às LER/Dort: o caso das indústrias de abate e processamento de carnes. Univ Psychol. 2013;2(4):1195-1209.

24. Ramos MZ, Bianchessi DLZ, Merlo ALC, Poersch AL, Veeck C, Heisller SZ, et al. Trabalho, adoecimento e histórias de vida em trabalhadoras da indústria calçadista. Estud psicol. 2010;15(2):207-15.

25. Ramos MZ. Trabalho, subjetividade e reabilitação profissional: por uma genealogia dos modos de vida [dissertação]. Porto Alegre: Universidade Federal do Rio Grande do Sul; 2005.

26. Scussiato LA, Céspedes LDM, Sarquis LMM, Stein AV Jr, Miranda FMA. Análise dos agravos relacionados ao trabalho notificados pela Unidade Saúde do Trabalhador. REME - Rev Min Enferm. 2010;14(1):88-95.

27. Cavalcante CAA, Santos SS, Cavalcante EFO, Martins RL, Silveira EA, et al. Perfil dos agravos relacionados ao trabalho notificados no Rio Grande do Norte, 2007 a 2009. Epidemiol Serv Saúde. 2014;23(4):741-52. 